ICRES 2019: International Conference on Robot

Ethics and Standards, London, UK, 29-30 July 2019.

https://doi.org/10.13180/icres.2019.29-30.07.009

\title{
EVALUATING THE USE OF HUMAN AWARE NAVIGATION IN INDUSTRIAL ROBOT ARMS
}

\author{
MATTHEW STORY, CYRIL JAKSIC, SARAH R. FLETCHER and PHILIP WEBB \\ School of Aerospace, Transport and Manufacturing, Cranfield University, College Rd, Wharley End, \\ Bedford, MK43 OAL, United Kingdom \\ E-mail:m.story@cranfield.ac.uk,cyril.jaksic@cranfield.ac.uk,s.fletcher@cranfield.ac.uk, \\ p.f.webb@cranfield.ac.uk \\ www.cranfield.ac.uk \\ JONATHAN CARBERRY \\ BAE Systems, Samlesbury Aerodrome, Building S609, Myerscough Smithy Rd, Balderstone, Blackburn \\ BB2 $7 L F$ \\ jon.carberry@baesystems.com
}

\begin{abstract}
Whilst the principles followed by modern standards for interaction between humans and robots follows the First Law of Robotics popularised in science fiction in the 1960's, the current standards regulating the interaction between humans and robots emphasise the importance of physical safety. However, they are less developed in another key dimension: psychological safety. As sales of industrial robots have been increasing over recent years, so has the frequency of Human-Robot Interaction (HRI). The present paper looks at the current safety guidelines for HRI in an industrial setting and assesses their suitability. The paper then presents a means to improve current standards utilising lessons learned from studies into Human Aware Navigation, which has seen increasing use in mobile robotics. The paper highlights the importance of considering a human not only as an object whose position needs to be refreshed, but to incorporate knowledge of human cognition in order to predict human behaviour. To understand this, it is necessary to focus less on how a robot avoids humans and more on how humans react when a robot is within the same space. Currently, the safety guidelines are behind the technological advance, however, with further studies aimed at understanding HRI and applying it to newly developed pathfinding and obstacle avoidance methods, interaction can move closer to cooperation.
\end{abstract}

\section{Introduction}

"A robot may not injure a human being, or, through inaction, allow a human being to come to harm", the First Law of Robotics put forward by Isaac Asimov ${ }^{1}$ is a guiding principle within Human-Robot Interaction (HRI). The interpretation of harm has primarily been interpreted as physical, despite Asimov not limiting the definition to that within his short stories. For instance, in pp.101 - 122, a robot gives people false information to prevent them from feeling upset in order to not break the first law. Currently, technology does not allow for a robot to be able to read someone's thoughts, but there is the technology and research available to understand how a robot's actions may affect a person's psychological well-being. This is becoming more relevant as the number of robots within the industrial environment increases. $^{2}$

Initially designed to be used as tools for completing highly repetitive tasks, ${ }^{3}$ the range of applications for robots has expanded significantly from providing meals, laundry, and basic patient care in hospitals, ${ }^{4}$ to tour guides in museums. ${ }^{5}$ High speed, high levels of repeatability, and continuous programming are all advantages that robots have over human workers. This is offset by the difficulty robots have adapting to dynamic changes in the working environment, which human workers can generally take in their stride.

In Ref. 6, it was highlighted that there are two elements of safety to be considered during HRI; physical safety (the action does not result in an injury for the human), and 
psychological safety (the action does not result in fear or surprise for the human). Research into navigation whilst maintaining psychological safety has been more limited in relation to industrial robot arms (stationary and typically high payload capabilities) than with mobile robotics (a robot capable of moving around the environment). Although mobile robots have been deployed for use in HRI since the late $1990 \mathrm{~s},{ }^{7}$ and the psychological effects of robotic behaviour were becoming prominent in the early $2000 \mathrm{~s}^{8}$ it was not until the mid-2000s that these were combined to design a path planner that would incorporate them both into what was coined Human Aware Navigation. ${ }^{9}$ Therefore, in order to assess the current state of Human Aware Navigation in industrial robot arms the following questions will be addressed:

- Can the current Human-Robot Interaction safety guidelines be optimised for maintaining the physical and psychological safety of the operator?

- Can the approaches for Human Aware Navigation in mobile robotics be applied to an industrial robot arm?

\section{Safety with Barriers - The Current State of Safety in Industrial Robot Arms}

With an increasing prevalence of HRI in an industrial environment, more attention has been recently paid to dynamic obstacle avoidance in static base robot arms. This research differs from mobile robot navigation in that the dynamic obstacles are generally assumed to be a person, and therefore, the path planning and obstacle avoidance are designed with physical safety as the main priority and come with a more conservative approach. ${ }^{10}$ The traditional method was the use of physical barriers to completely enclose the robot when it is in operation. However, this is beginning to change as shown by the guidelines set in ISO $10218^{11}$ and ISO/TS $15066 .{ }^{12}$ Following these guidelines, when a human enters the workspace of the robot arm, one of the three following measures must take place for safe collaborative operation.

The first measure is called a Safety-rated monitored stop which involves the robot ceasing motion before the operator enters within a pre-set distance from the robot, and may resume once the operator has left. The second measure is called Speed and Separation monitoring which involves the robot maintaining a protective separation distance, which can be reduced with reduced robot speed. If the distance is reduced to below a set value, then the robot ceases motion. A third measure is called Power and Force limiting, it involves reducing the levels of impact should a physical contact between the robot and the operator occur. The reduced impact can be achieved by increasing the contact surface area, mechanisms and/or materials for absorbing the energy, extending energy transfer time, and limiting movement masses. These can be categorised into two distinct strategies: Pre-collision and Post-collision. A pre-collision strategy aims to prevent a collision from happening (Safetyrated monitored stop, Speed and Separation monitoring), whilst a post-collision strategy aims to minimise the potential damage when a collision occurs (Power and Force limiting). Although not a collision avoidance/mitigation strategy, ISO/TS 15066 also mentions Hand Guiding within the HRI guidelines, which involves the robot performing a Safety-rated monitored stop followed by the operator manoeuvring the end effector.

Although the collision strategies significantly reduce the physical harm, they do not fully mitigate the likelihood of an injury. ${ }^{13}$ A person can still collide with inanimate objects, which means that the robot stopping when a person enters the workspace is not a guarantee of physical safety, and even with the robot's speed and force being reduced, the person can still come to harm as a result of their speed and force. Furthermore, a robot that would either collide with or ceases working when a person enters the workspace is not ideal for HRI. The strategies also do not consider the effects of HRI on the person's mental strain, which has 
been linked to the actions taken by the robot. ${ }^{14}$

The strategies have also not taken into account technological advances, such as the development of increasingly complex on-board systems, especially machine vision systems. Whereas early robotic systems were reliant on laser distance scanners for interpreting the world around them, and many are still used, more modern systems can use depth and colour camera systems capable of relaying a significantly greater amount of information. ${ }^{15,16}$ A laser scanner can provide a highly reactive and detailed image of the distance of an object from the robot, but it cannot be used to interpret human features, gestures, or emotions in the same way as an RGB-D camera. Furthermore, the increase of processing power allows for detailed analysis of what a vision system is receiving. Therefore, the capabilities of robots in HRI have improved in both complexity of the tasks and ability to keep the operator safe, as well as receive and interpret information regarding the person's stress and mental workload.

By taking the factors mentioned above into consideration, one can argue that the current guidelines are not optimal for maintaining the physical and psychological safety of the operator. Human Aware Navigation,${ }^{9}$ a field of mobile robotics, does take both physical and psychological safety into consideration. The lessons learned from studies into this recent field may provide a means to inform and improve the current guidelines.

\section{Human Aware Navigation}

In order to operate and be accepted in the same environment as people, a robot must not only be able to avoid collisions with them, but also recognise and act accordingly to the social behaviour of humans. ${ }^{17}$ Pathfinding and avoidance algorithms that take this factor into account are now finding increased relevance in robotics. ${ }^{18}$ Ref. 19 argued that for navigation to be considered human-aware, the robot should be able to convey in an understandable manner its current state, current goal, as well as its imminent move. Since this definition in 2000, the criteria for Human Aware Navigation have become more sophisticated. In this section, studies using the concepts of Human Aware Navigation will be reviewed, first for mobile robotics and then for robot arms, assessing similarities to determine if the former can inform the latter.

\subsection{Mobile Robots}

For Human Aware Navigation, the robot must be able to make decisions which ensure not only safe, reliable, and effective motion, but also socially acceptable motions. ${ }^{20}$ Ref. 21 states that proxemics provide the fundamental outline for a socially acceptable distance for people. This can then be utilised as a reference for socially acceptable distances for robots. These socially acceptable distances can be used to designate comfort zones, with the closer the zone, the higher levels of stress the person would experience should a stranger enter it. However, there is no consensus on the most accurate social spacing model. The most prominent models for social spacing around an individual can be found in Ref. 22 . The model displays four social spacing shapes around a person which dictate the different comfort zones; concentric circles, egg-shaped, elliptical, and elliptical which is skewed on the dominant side. Each of these shapes can be applied depending on the context of the interaction, but they all suggest that the social sensitivity of the person decreases with increasing distance away from the person.

Ref. 23,24 used such a model to develop an algorithm leading the robot to avoid an elliptical space, deemed too close for comfort, outside of one's field of view. In this condition, the robot followed a smooth path at a socially acceptable distance around the person. When compared to a static obstacle avoidance algorithm, which does not apply social distances, there was an increase in physical comfort. An advantage to designing a framework using 
Human Aware Navigation is that it does not require a complete overhaul of existing frameworks for maintaining physical safety. Ref. 25,26 also applied a proxemics based model when developing their mobile robots, which reduced the stress caused to a person as a result of the robot navigating a crowd. Ref. 27 iterated on their design further by adding the capability of predicting the person's reaction to the robot's possible actions and taking the course with the lowest social work impact, again reducing the stress further. Ref. 28 expanded on this model to develop a socially acceptable, human-like collision avoidance system for a robot moving amongst pedestrians. As with Ref. 27, the model was first calibrated by the robot moving towards the person without collision avoidance to determine the socially acceptable distance instead of using proxemics. The system also operated on a collaborative avoidance basis, where both parties move to avoid the collision, as is the case in most human-human collision avoidance situations. ${ }^{29}$ Surveys taken by participants reported that the robot with the updated model gave the perception of being safer, as well as the results showing the avoidance system performed objectively safer.

Although the human always having priority is generally considered an important aspect for social path planning, ${ }^{30}$ people unfamiliar with a robot and its capabilities will usually opt to give way. ${ }^{29}$ This highlights another issue regarding how the robot can convey it's current goal. Ref. 31,32 proposed five methods for a robot to express intent without the use of verbal or facial clues. Sound was shown to be the most legible method to indicate approach intention when within the intimate zone $(<0.46 \mathrm{~m})$, and approach angle (orientation, posture, and body movement) was significantly better further away than the intimate zone. The participants were more comfortable when the robot was approaching at an angle instead of head on, a similar finding to Ref. 33 previously. Ref. 34 iterated further to develop the requirements for a proxemically competent robot.

In HRI, the robot must also be able to interpret a person's intent. A person's orientation and body pose with respect to the objects around them is a key social cue necessary for a robot to operate within the same environment. Without this, a robot would fail in a task as commonplace as standing in a queue. ${ }^{17}$ The body pose can be a useful indicator of the short-term motions a person will make, and face orientation has the potential to show a person's intent in the long term. ${ }^{35}$ Furthermore, face orientation can provide cues as to a person's field of view. Ref. 36 developed a model taking into consideration not only the persons perceived field of vision but also potential sources of occlusion. This was designed to reduce the chances of the robot to surprise the person, but can also be applied to ensure the person can clearly see the robot and displays of planned movement.

The speed of the robot has also been shown to affect a person's comfort. Ref. 8 used a mobile robot at varying speeds when approaching a person. The speeds for optimal comfort were between $0.25-0.4 \mathrm{~m} / \mathrm{s}$, but significant discomfort was not reported until $1.0 \mathrm{~m} / \mathrm{s}$, whilst discomfort and frustration were suggested at speeds below $0.25 \mathrm{~m} / \mathrm{s}$. The frustration at low speeds and preference for higher speeds by the people is further corroborated by Ref. 37 for a robot using collision avoidance. This was attributed to the robot avoiding a potential collision quicker at higher speeds, and frustration at the delay in avoidance caused by lower speeds. The robot was also perceived as less safe at lower speeds. Though these studies found similar results, they all used robots of a similar height (between waist and head height for an average person). Little is known as to how the height and size of the robot would effect speed on a person's comfort.

From this review into Human Aware Navigation in mobile robotics the path planner can utilise a model based upon proxemics spacing. However, the distances for what is socially acceptable require updating for HRI, as these have been shown to differ when compared to human-human interaction. A model incorporating an understanding of a person's available field of view has also been shown to help improve the interaction. Table.1 gives a summary 
of the main findings in section 3.1 .

Table 1. A summary of the lessons learned from reviewing the current standards of Human Aware Navigation in Mobile Robotics

\begin{tabular}{cc}
\hline $\begin{array}{c}\text { Studied and understood } \\
\text { in Mobile Robots }\end{array}$ & $\begin{array}{c}\text { Requires more research to be } \\
\text { confidently understood in Mobile Robots }\end{array}$ \\
\hline $\begin{array}{c}\text { Proxemic guidelines for human to human interaction } \\
\text { can be applied to mobile robotics }\end{array}$ & $\begin{array}{c}\text { Link between robot's size/shape and } \\
\text { person's comfort }\end{array}$ \\
$\begin{array}{c}\text { Human Aware Navigation can be used alongside } \\
\text { existing navigation methods }\end{array}$ & $\begin{array}{c}\text { The appropriate proxemic spacing model } \\
\text { for a mobile robot }\end{array}$ \\
Robot legibility increases the person's comfort & $\begin{array}{c}\text { How does the robot's speed and proximity } \\
\text { effect the person's comfort }\end{array}$ \\
\hline
\end{tabular}

\subsection{Robot Arms}

Unlike physical safety maintenance, the development of Human Aware Navigation in robot arms is less developed than in mobile robotics. This could be due to the robot arms being more applied in industry, where they are often separated by physical barriers. Such a setting greatly limits the opportunity for HRI and, as a result, reduces the relevance of psychological safety considerations. Once the barriers are removed however, robot arms should be meeting the same psychological safety measures as mobile robots. Because the removal of the barriers and the introduction of collaborative robots is becoming more prominent, psychological safety with robot arms is more relevant than ever.

Although robot arms and mobile robots share many qualities, there are some significant differences. One would be the extra dimension of available movement and the added degrees of freedom of movement in robot arms. This inherently makes the robot arms more complex, making its planned movements harder to read. ${ }^{38}$ Proximity and speed of the robot arm during HRI are key measures. A decreased distance increases the mental workload and discomfort of a person, but the effects of speed are less clear. ${ }^{39,40}$ Similar to mobile robotics, the influence of size of the robot on comfort and mental workload are not clearly established.

By improving the psychological safety of a robot, there is a desirable side effect. A robot arm utilising Human Aware Navigation, generally increases the operator's comfort, improving their efficiency ${ }^{14}$ and also the efficiency of the robot as it will have less idle time. ${ }^{41}$ The reduced idle time may be attributed to the implementation of path predictive planners, a key part of Human Aware Navigation. Therefore, rather than reacting to the sudden appearance of the person and waiting until they have vacated to a safe distance, the robot can adapt and move around to prevent the emergency stop taking place. By accurately predicting where the person will be in accordance with their own position, the robot can also reduce annoyance, surprise or obstruction. ${ }^{9}$ Despite the perceived improvements in comfort levels experienced by people when a robot is using Human Aware Navigation, Ref. 14 highlights that their study and similar previous studies only observe these changes for a relatively short period of time. As the end goal of many studies is for a system to be implemented in an industrial environment, the robots would be collaborating with human workers for an extended period of time which may present unforeseen variables.

In industrial HRI, overall trust in the robot is linked to trust in the robot completing the task. Ref. 42 assessed the extent to which HRI task efficiency is dependent on how much the human trusted the robot with the task. The results showed that task efficiency did improve as trust increased up to a point, from which an over-reliance in the robot would 
then decrease performance. This would suggest that there is an optimal level of trust over which performance would be impaired.

As with physical safety only pathfinding and obstacle avoidance, mobile robots and robot arms can follow similar principles, with some minor changes due to the way they move throughout the world and the different applications they have. Path predictive planners, speed and proximity are all measures that can be transferred across from mobile robots to robot arms with modification. Nevertheless, the impact these have on the person during HRI requires further study. The data from these studies can then be implemented into a Human Aware Navigation algorithm for a robot arm, and also aid in developing improved safety guidelines for robot arms in industrial HRI.

\section{Conclusion}

At the beginning of this review, a question was posed: Can the current Human-Robot Interaction safety guidelines be optimised for maintaining the safety of the operator? The safety guidelines set in TS 15066 present methods of reducing the likelihood of physical injury as a result of a robot's actions but, as highlighted in the introduction of this paper, that is considering only the potential physical impact in HRI. Therefore, they can be improved upon. In order to improve the quality of the human element of Human-Robot Interaction, it is important to develop a better understanding of how the robot's action (or inaction) can influence the operator. A solution to this was proposed by using the guidelines which inform Human Aware Navigation.

Therefore, a second question was posed: Can the approaches for Human Aware Navigation in mobile robotics be applied to an industrial robot arms? The reviews in sections 3.1 and 3.2 show the potential crossover between human aware planners in mobile robotics and in robot arms and is summarised in Table.2.

Table 2. A summary of the lessons learned from Human Aware Navigation in mobile robotics and their potential applications in industrial robot arms

\begin{tabular}{|c|c|}
\hline $\begin{array}{l}\text { Can be readily transferred } \\
\text { from Mobile Robots to Industrial Robot Arms }\end{array}$ & $\begin{array}{l}\text { Requires more research before } \\
\text { being applied to Industrial Robot Arms }\end{array}$ \\
\hline $\begin{array}{l}\text { Interpretation of a person's intent by the } \\
\text { robot through machine vision and learning }\end{array}$ & Legible and predictable robot motion \\
\hline \multirow[t]{3}{*}{$\begin{array}{l}\text { Robot motion based upon a person's field } \\
\text { of view }\end{array}$} & $\begin{array}{c}\text { How does the robot's speed and proximity } \\
\text { affect the person's comfort }\end{array}$ \\
\hline & $\begin{array}{c}\text { Link between the robot's size/shape and } \\
\text { the person's comfort }\end{array}$ \\
\hline & $\begin{array}{l}\text { The appropriate proxemic spacing model } \\
\text { for an industrial robot arm }\end{array}$ \\
\hline
\end{tabular}

Despite the limitations of studies into Human Aware Navigation and the difficulties of the subjective nature of concepts such as comfort, fear, and surprise, it is clear that speed and proximity of a robot can effect a person's comfort. This can lead to an objective improvement when using Human Aware Navigation in HRI: the efficiency of both the human and the robot is increased. Even with this improved efficiency, HRI is not widespread in industry. One of the main reasons for the relatively few occurrences of such interaction can be attributed to safety regulations being behind the advances in technology. Nevertheless, with further studies and research into Human Aware Navigation, which highlight the advantages mentioned in 3.2 , as well as the significant improvements in robotics safety without the requirement of 
physical barriers, this is due for change. The current guidelines operate from an objective viewpoint, humans do not.

The first steps into achieving this would be to further assess how an industrial robot arm's speed and proximity effect an operator's mental workload and comfort whilst working collaboratively with the robot, when the robot is utilising a human avoidance algorithm. The study will benefit from assessing if there is a significant change on the operator's mental workload and comfort with a robot of larger size and payload. This data can then be used to further inform and improve current safety guidelines and Human Aware Navigation in industrial robot arms. By furthering our knowledge of this key aspect in HRI, the acceptance of industrial robot arms in a shared workspace can be considered that much closer.

\section{References}

1. I. Asimov, I, Robot (Dobson Books Ltd, Great Britain, 1967).

2. Executive Summary World Robotics 2018 Industrial Robots, tech. rep., International Federation of Robotics (2018).

3. G. Charalambous, S. R. Fletcher and P. Webb, Int. J. Adv. Manuf. Technol 91, 2465 (2017).

4. R. Bloss, IND ROBOT 43, 463 (2016).

5. S. Thrun, IEEE Intell. Syst 13, 27 (1998).

6. S. Nonaka, K. Inoue, T. Arai and Y. Mae, Evaluation of human sense of security for coexisting robots using virtual reality. 1st report: evaluation of pick and place motion of humanoid robots, in IEEE International Conference on Robotics and Automation, 2004. Proceedings. ICRA '04, (New Orleans, LA, USA, 2004).

7. W. Burgard, A. Cremers and D. Fox, The interactive museum tour-guide robot, in Aaai/iaai, (Madison, Wisconsin, USA, 1998).

8. J. T. Butler and A. Agah, Auton Robots 10, 185 (2001).

9. E. A. Sisbot, L. F. Marin-Urias, R. Alami and T. Simeon, IEEE Transactions on Robotics 23, 874 (2007).

10. F. Flacco, T. Kroger, A. D. Luca and O. Khatib, A depth space approach to human-robot collision avoidance, in Robotics and Automation (ICRA), 2012 IEEE International Conference, (Saint Paul, Minnesota, USA, 2012).

11. Robots and robotic devices - Safety requirements for industrial robots. Part 2: Robot systems and integration (ISO 10218:2011), standard, International Standards Organisation (Geneva, Switzerland, 2011).

12. Robots and robotic devices - Collaborative robots (ISO/TS 15066:2016), standard, International Standards Organisation (Geneva, Switzerland, 2016).

13. P. Zhang, P. Jin, G. Du and X. Liu, IND. ROBOT 43, 264 (2016).

14. P. A. Lasota and J. A. Shah, Human Factors 57, 21 (2015).

15. J. U. Kühnle, Z. Xue, M. Stotz, J. M. Zöllner, A. Verl and R. Dillmann, Grasping in depth maps of time-of-flight cameras, in 2008 International Workshop on Robotic and Sensors Environments, (Ottawa, ON, Canada, 2008).

16. Q. Wang, G. Kurillo, F. Ofli and R. Bajcsy, Evaluation of pose tracking accuracy in the first and second generations of microsoft kinect, in Proceedings - 2015 IEEE International Conference on Healthcare Informatics, ICHI 2015, (Dallas, TX, USA, 2015).

17. Y. Nakauchi and R. Simmons, Auton Robots 12, 313 (2002).

18. K. Charalampous, I. Kostavelis and A. Gasteratos, Rob Auton Syst 93, 85 (2017).

19. R. Alami, I. Belousov, S. Fleury, M. Herrb, F. Ingrand, J. Minguez and B. Morisset, Diligent: Towards a human-friendly navigation system, in IEEE International Conference on Intelligent Robots and Systems, (Takamatsu, Japan, 2000).

20. E. A. Sisbot, R. Alami, T. Simeon, K. Dautenhahn, M. Walters, S. Woods, K. L. Koay and C. Nehaniv, Navigation in the presence of humans, in 5th IEEE-RAS International Conference on Humanoid Robots, 2005, (Tsukuba, Japan, 2005).

21. E. T. Hall, The Hidden Dimension: Man's Use of Space in Public and Private (The Bodley Head, London, 1966).

22. P. Papadakis, P. Rives and A. Spalanzani, Adaptive spacing in human-robot interactions, in Intelligent Robots and Systems (IROS 2014), 2014 IEEE/RSJ International Conference on, (Chicago, USA, 2014). 
23. A. K. Pandey and R. Alami, A framework for adapting social conventions in a mobile robot motion in human-centered environment, in 2009 International Conference on Advanced Robotics, (Munich, Germany, 2009).

24. A. K. Pandey and R. Alami, A framework towards a socially aware mobile robot motion in human-centered dynamic environment, in IEEE/RSJ 2010 International Conference on Intelligent Robots and Systems, IROS 2010 - Conference Proceedings, (Taipei, Taiwan, 2010).

25. G. Ferrer, A. Garrell and A. Sanfeliu, Robot companion: A social-force based approach with human awareness-navigation in crowded environments, in IEEE International Conference on Intelligent Robots and Systems, (Tokyo, Japan, 2013).

26. G. Ferrer, A. Garrell and A. Sanfeliu, Social-aware robot navigation in urban environments, in 2013 European Conference on Mobile Robots, ECMR 2013 - Conference Proceedings, (Barcelona, Spain, 2013).

27. G. Ferrer and A. Sanfeliu, Proactive kinodynamic planning using the extended social force model and human motion prediction in urban environments, in Intelligent Robots and Systems (IROS 2014), 2014 IEEE/RSJ International Conference on, (Chicago, IL, USA, 2014).

28. M. Shiomi, F. Zanlungo, K. Hayashi and T. Kanda, Int. J. Soc. Robot 6, 443 (2014).

29. C. Vassallo, A. H. Olivier, P. Souères, A. Crétual, O. Stasse and J. Pettré, Gait and Posture 51, 97 (2017)

30. J. V. Gómez, N. Mavridis and S. Garrido, Social path planning: Generic human-robot interaction framework for robotic navigation tasks, in 2nd Intl. Workshop on Cognitive Robotics Systems: Replicating Human Actions and Activities, (Tokyo, Japan, 2013).

31. C. L. Bethel and R. R. Murphy, HRI 6, 327 (2006)

32. C. L. Bethel and R. R. Murphy, Non-facial/non-verbal methods of affective expression as applied to robot-assisted victim assessment, in Proceeding of the ACM/IEEE international conference on Human-robot interaction - HRI '07, (Arlington, VA, USA, 2007).

33. K. Dautenhahn, M. Walters, S. Woods, K. L. Koay, C. L. Nehaniv, A. Sisbot, R. Alami, T. Siméon, C. Lane and E. A. Sisbot, How may i serve you ? a robot companion approaching a seated person in a helping context, in Proceedings of the 1st ACM SIGCHI/SIGART conference on Human-robot interaction, (Salt Lake City, Utah, USA, 2006).

34. Z. Henkel, R. Murphy, C. Bethel and V. Srinivasan, A proxemic-based hri testbed, in Performance Metrics for Intelligent Systems (PerMIS) Workshop, (College Park, Maryland, USA, 2016).

35. P. Ratsamee, Y. Mae, K. Kamiyama, M. Horade, M. Kojima and T. Arai, ROBOMECH 2, p. $11(2015)$.

36. E. A. Sisbot, L. F. Marin, R. Alami and T. Simeon, A mobile robot that performs human acceptable motions, in IEEE International Conference on Intelligent Robots and Systems, (Beijing, China, 2006).

37. E. Pacchierotti, H. I. Christensen and P. Jensfelt, Human-robot embodied interaction in hallway settings: a pilot user study, in ROMAN 2005. IEEE International Workshop on Robot and Human Interactive Communication, 2005., (Nashville, TN, USA, 2005).

38. H. Kozima, M. P. Michalowski and C. Nakagawa, Int. J. Soc. Robot 1, 3 (2009).

39. J. Stark, R. R. C. Mota and E. Sharlin, Personal space intrusion in human-robot collaboration, in Companion of the 2018 ACM/IEEE International Conference on Human-Robot Interaction, (Chicago, IL, USA, 2018).

40. J. T. C. Tan, F. Duan, Y. Zhang, K. Watanabe, R. Kato and T. Arai, Human-robot collaboration in cellular manufacturing: Design and development, in 2009 IEEE/RSJ International Conference on Intelligent Robots and Systems, (St. Louis, MO, USA, 2009).

41. V. V. Unhelkar, P. A. Lasota, Q. Tyroller, R.-D. Buhai, L. Marceau, B. Deml and J. A. Shah, IEEE RA-L 3, 2394 (2018).

42. M. Chen, S. Nikolaidis, H. Soh, D. Hsu and S. Srinivasa, Pull-in time dynamics as a measure of absolute pressure, in Proceedings of the 2018 ACM/IEEE International Conference on HumanRobot Interaction, (Chicago, IL, USA, 2018).

\section{Acknowledgements}

The research is funded by EPSRC Centre for Innovative Manufacturing and Intelligent Automation and BAE Systems. 